\title{
Cooling of Neutron Stars
}

\author{
Hovik Grigorian \\ JINR Dubna, Russia
}

\begin{abstract}
We introduce the theoretical basis for modeling the cooling evolution of compact stars starting from Boltzmann equations in curved space-time. We open a discussion on observational verification of different neutron star models by consistent statistics. Particular interest has the question of existence of quark matter deep inside of compact object, which has to have a specific influence on the cooling history of the star. Besides of consideration of several constraints and features of cooling evolution, which are susceptible of being critical for internal structure of hot compact stars we have introduced a method of extraction of the mass distribution of the neutron stars from temperature and age data. The resulting mass distribution has been compared with the one suggested by supernove simulations. This method can be considered as an additional checking tool for the consistency of theoretical modeling of neutron stars. We conclude that the cooling data allowed existence of neutron stars with quark cores even with one-flavor quark matter.
\end{abstract}

\section{Acknowledgements}

I thank the organizers of the Helmholtz International Summer School on "Dense Matter in Heavy-Ion Collisions and Astrophysics" at JINR Dubna providing me the opportunity to participate in the school. I am grateful to D. Blaschke for useful comments and discussions. I also acknowledge all my coauthors for our collaboration.

\section{References}

1. H. Grigorian, Phys. Part. Nucl. 39 (2008) 1143

2. T. Klahn et al., Phys. Lett. B 654, 170 (2007)

3. J. M. Lattimer and M. Prakash, Astrophys. J. 550, 426 (2001)

4. D. Blaschke, S. Fredriksson, H. Grigorian, A. M. Oztas and F. Sandin, Phys. Rev. D 72, 065020 (2005)

5. D. Blaschke, H. Grigorian and D. N. Voskresensky, Astron. Astrophys. 424, 979 (2004)

6. D. Page, U. Geppert and F. Weber, Nucl. Phys. A 777, 497 (2006)

7. T. Takatsuka and R. Tamagaki, Prog. Theor. Phys. 112, 37 (2004)

8. S. Popov, H. Grigorian and D. Blaschke, Phys. Rev. C 74, 025803 (2006)

9. T. Schafer, Phys. Rev. D 62, 094007 (2000)

10. H. Grigorian, Phys. Rev. C 74, 025801 (2006)

11. M. Prakash, I. Bombaci, M. Prakash, P. J. Ellis, J. M. Lattimer and R. Knorren, Phys. Rept. 280, 1 (1997)

12. D. Blaschke and H. Grigorian, Prog. Part. Nucl. Phys. 59, 139 (2007)

13. Haensel, P., Potekhin, A.Y., Yakovlev, D.G Neutron Stars 1: Equation of State and Structure (Springer, 2007) $619 \mathrm{p}$.
14. Weber F. Pulsars as Astrophysical Laboratories for Nuclear and Particle Physics (IoP Publishing, 1999) $682 \mathrm{p}$.

15. Shapiro S.L., Teukolsky S.A. Black holes, white dwarfs, and neutron stars (Wiley VCH, USA, 1983) $672 \mathrm{p}$.

This is an Open Access article distributed under the terms of the Creative Commons Attribution-Noncommercial License 3.0, which permits unrestricted use, distribution, and reproduction in any noncommercial medium, provided the original work is properly cited. 


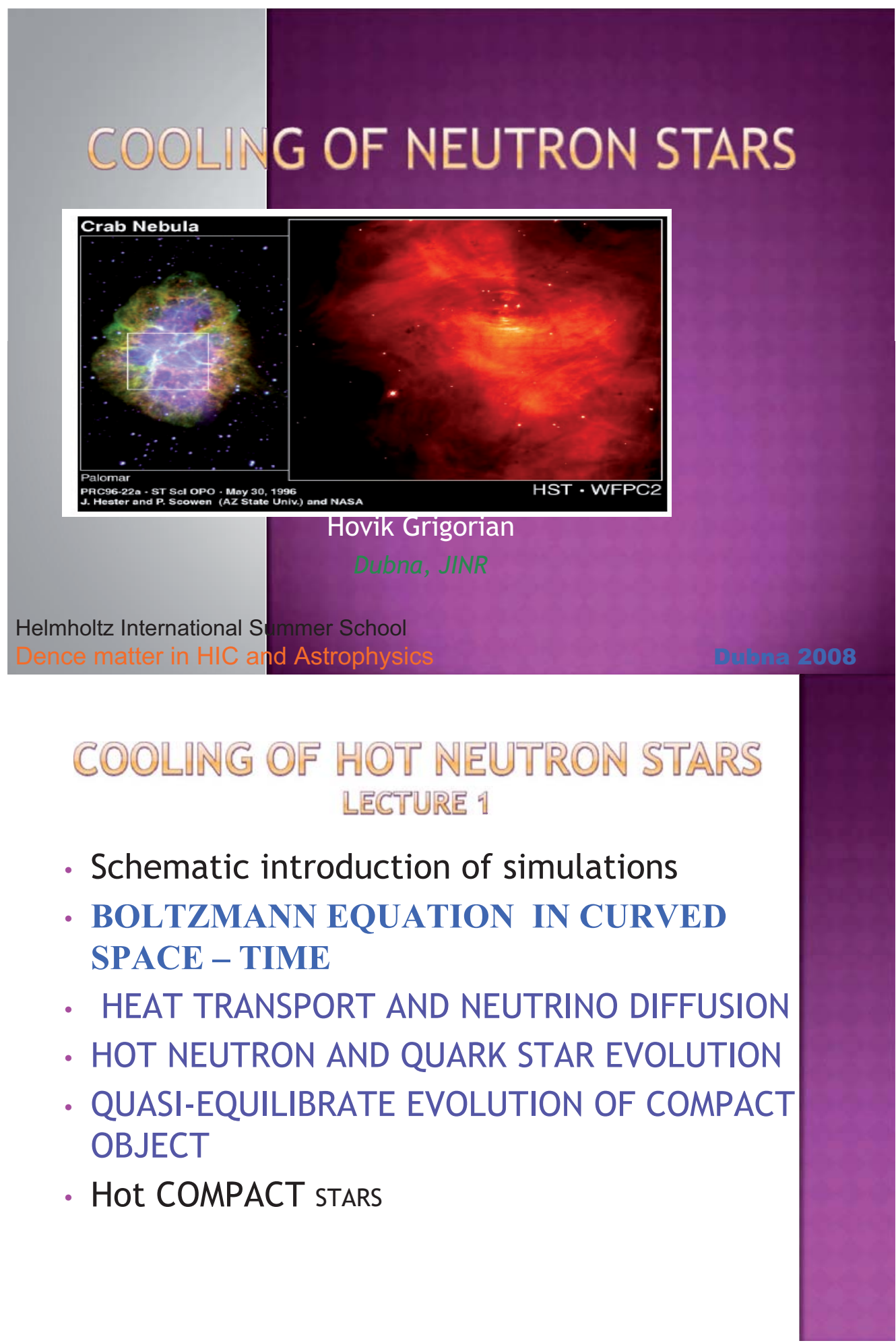




\section{PHASE DIAGRAM \& COOLING SIAULATIIONS}
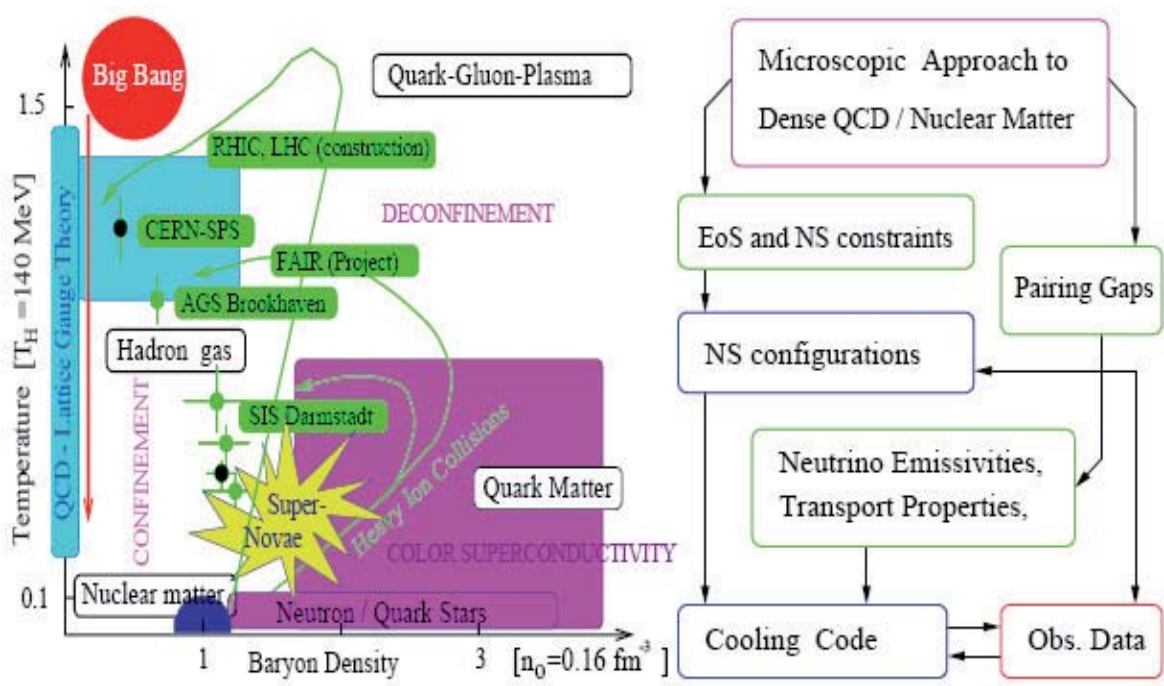

\section{BOLTZMANN EQUATION IN CURVED SPACE - TIME}

The Boltzmann equation for massless particles

Lindquist, R.W. 1966, Ann. Phys., 37, 478

$$
p^{\beta}\left(\frac{\partial f}{\partial x^{\beta}}-\Gamma_{\beta \gamma}^{\alpha} p^{\gamma} \frac{\partial f}{\partial p^{\alpha}}\right)=\left(\frac{d f}{d \tau}\right)_{\text {coll }}
$$

- $f$ is the invariant neutrino distribution function

- $p^{a}$ is the neutrino 4-momentum

- $\Gamma_{\beta \gamma}^{\alpha}$ are the Christoffel symbols for the metric

$$
d s^{2}=-e^{2 \phi} d t^{2}+e^{2 \Lambda} d r^{2}+r^{2} d \theta^{2}+r^{2} \sin ^{2} \theta d \Phi^{2}
$$

The BE in the comoving basis

$$
p^{b}\left(e_{b}^{\beta} \frac{\partial f}{\partial x^{\beta}}-\Gamma_{b c}^{a} p^{c} \frac{\partial f}{\partial p^{a}}\right)=\left(\frac{d f}{d \tau}\right)_{c o l l}
$$

The non-zero Ricci coefficients are

$$
\begin{aligned}
& \Gamma_{00}^{1}=\Gamma_{01}^{0}=e^{-\phi} e^{-\Lambda}\left(\frac{\partial\left(\gamma e^{\phi}\right)}{\partial r}+\frac{\partial\left(\gamma v e^{\Lambda}\right)}{\partial t}\right), \quad \Gamma_{11}^{0}=\Gamma_{10}^{1}=e^{-\phi} e^{-\Lambda}\left(\frac{\partial\left(\gamma e^{\Lambda}\right)}{\partial t}+\frac{\partial\left(\gamma v e^{\phi}\right)}{\partial r}\right), \\
& \Gamma_{20}^{2}=\Gamma_{22}^{0}=\Gamma_{30}^{3}=\Gamma_{33}^{0}=-v \Gamma_{22}^{1}=v \Gamma_{21}^{2}=-v \Gamma_{33}^{1}=v \Gamma_{31}^{3}=\frac{\gamma v e^{-\Lambda}}{r}, \Gamma_{33}^{2}=-\Gamma_{32}^{3}=-\frac{\cot \theta}{r} .
\end{aligned}
$$




\section{HEAT TRANSPORT AND NEUTRINO DIFFUSION}

The neutrino 4-momentum is

$$
p^{a}=\left(\omega, \omega \mu, \omega\left(1-\mu^{2}\right)^{1 / 2} \cos \Phi, \omega\left(1-\mu^{2}\right)^{1 / 2} \sin \Phi\right)
$$

- $\mu$ is the cosine of the angle between the neutrino momentum and the radial direction

- $\omega$ is the neutrino energy in a comoving frame

The BE in the spherically symmetric case

$$
\begin{aligned}
\omega\left(e_{0}^{t}+\mu e_{1}^{t}\right) \frac{\partial f}{\partial t} & +\omega\left(e_{0}^{r}+\mu e_{1}^{r}\right) \frac{\partial f}{\partial r}-\omega^{2}\left(\mu \Gamma_{00}^{1}+\mu^{2} \Gamma_{10}^{1}+\left(1-\mu^{2}\right) \Gamma_{20}^{2}\right) \frac{\partial f}{\partial \omega} \\
& -\omega\left(1-\mu^{2}\right)\left(\Gamma_{00}^{1}+\Gamma_{22}^{1}+\mu \Gamma_{10}^{1}-\mu \Gamma_{20}^{2}\right) \frac{\partial f}{\partial \mu}=\left(\frac{d f}{d \tau}\right)_{\text {coll }} .
\end{aligned}
$$

Applying the operator to equation (5) and defining the $i^{\text {th }}$ moment Thorne, K. S. 1981, MNRAS 194, 439

$$
\frac{1}{2} \int_{-1}^{+1} d \mu \mu^{i}, \quad i=0,1,2, \cdots, M_{i}=\frac{1}{2} \int_{-1}^{+1} d \mu \mu^{i} f, \quad Q_{i}=\frac{1}{2} \int_{-1}^{+1} d \mu \mu^{i}\left(\frac{d f}{d \tau}\right)_{\text {coll }} .
$$

\section{HEAT TRANSPORT AND NEUTRINO DIFFUSION}

Let us introduce $N_{\nu}, F_{\nu}$, and $S_{N}$ are the number density, number flux and number source term, respectively, while $J_{\nu}, H_{\nu}, P_{\nu}$, and $S_{E}$ are the neutrino energy density, energy flux, pressure, and the energy source term:

$$
\begin{gathered}
N_{\nu}=\int_{0}^{\infty} \frac{d \omega}{2 \pi^{2}} M_{0} \omega^{2}, \quad F_{\nu}=\int_{0}^{\infty} \frac{d \omega}{2 \pi^{2}} M_{1} \omega^{2}, \quad S_{N}=\int_{0}^{\infty} \frac{d \omega}{2 \pi^{2}} Q_{0} \omega \\
J_{\nu}=\int_{0}^{\infty} \frac{d \omega}{2 \pi^{2}} M_{0} \omega^{3}, \quad H_{\nu}=\int_{0}^{\infty} \frac{d \omega}{2 \pi^{2}} M_{1} \omega^{3}, \quad P_{\nu}=\int_{0}^{\infty} \frac{d \omega}{2 \pi^{2}} M_{2} \omega^{3}, \quad S_{E}=\int_{0}^{\infty} \frac{d \omega}{2 \pi^{2}} Q_{0} \omega^{2} .
\end{gathered}
$$

- integrating over the neutrino energy

- utilizing the continuity equation

- assumption of a quasi-static evolution

The neutrino transport equation Burrows, A., \& Lattimer, J. M. 1986, ApJ, 307, 178

$$
\begin{gathered}
\partial\left(N_{\nu} / n_{B}\right)+\begin{array}{c}
\partial\left(e^{\phi} 4 \pi r^{2} F_{\nu}\right) \\
\partial a
\end{array}=e^{\phi^{\phi} S_{N}} n_{B} \\
\frac{\partial\left(J_{\nu} / n_{B}\right)}{\partial t}+P_{\nu} \frac{\partial\left(1 / n_{B}\right)}{\partial t}+e^{-\phi} \frac{\partial\left(e^{2 \phi} 4 \pi r^{2} H_{\nu}\right)}{\partial a}=e^{\phi} \frac{S_{E}}{n_{B}}
\end{gathered}
$$




\section{HEAT TRANSPORT AND NEUTRINO DIFFUSION}

The distribution function in the diffusion approximation:

$$
f(\omega, \mu)=f_{0}(\omega)+\mu f_{1}(\omega), \quad f_{0}=\left[1+e^{\left(\begin{array}{c}
\omega-\mu_{\nu} \\
k T
\end{array}\right)}\right]^{-1}
$$

- $f_{0}$ is the distribution function at equilibrium $\left(T=T_{\text {mat }}, \mu_{\nu}=\mu_{\nu}^{e q}\right)$

- $\omega$ and $\mu_{\nu}$ are the neutrino energy and chemical potential

The moments $M_{i}$ of $f$ are

$$
M_{0}=f_{0}, M_{1}=\frac{1}{3} f_{1}, M_{2}=\frac{1}{3} f_{0}, \quad \text { and } \quad M_{3}=\frac{1}{5} f_{1} .
$$

The equation (??) is now

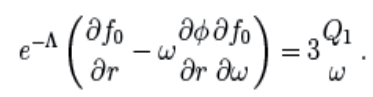

The collision term $Q_{1}$ can be represented as

$$
\left(\frac{d f}{d \tau}\right)_{\text {coll }}=\omega\left(j_{a}(1-f)-\frac{f}{\lambda_{a}}+j_{s}(1-f)-\frac{f}{\lambda_{s}}\right)
$$

- $j_{a}$ is the emissivity

- $\lambda_{a}$ is the absorptivity

- $j_{s}$ and $\lambda_{s}$ are the scattering contributions

\section{HEAT TRANSPORT AND NEUTRINO DIFFUSION}

The explicit form of the diffusion coefficient $D$

$$
D(\omega)=\left(j+\frac{1}{\lambda_{a}}+\kappa_{1}^{s}\right)^{-1} .
$$

Using the relation ( $\eta_{\nu}=\mu_{\nu} / T$ is the neutrino degeneracy parameter)

$$
\frac{\partial f_{0}}{\partial r}=-\left(T_{\partial r}^{\partial \eta_{\nu}}+\begin{array}{c}
\omega \partial T \\
T \partial r
\end{array}\right) \frac{\partial f_{0}}{\partial \omega},
$$

we obtain

$$
f_{1}=-D(\omega) e^{-\Lambda}\left[T \frac{\partial \eta}{\partial r}+\frac{\omega}{T e^{\phi}} \frac{\partial\left(T e^{\phi}\right)}{\partial r}\right]\left(-\frac{\partial f_{0}}{\partial \omega}\right) .
$$

Now the energy-integrated lepton and energy fluxes are

$$
\begin{aligned}
& F_{\nu}=-\frac{e^{-\Lambda} e^{-\phi} T^{2}}{6 \pi^{2}}\left[D_{3} \frac{\partial\left(T e^{\phi}\right)}{\partial r}+\left(T e^{\phi}\right) D_{2} \frac{\partial \eta}{\partial r}\right] \\
& H_{\nu}=-\frac{e^{-\Lambda} e^{-\phi} T^{3}}{6 \pi^{2}}\left[D_{4} \frac{\partial\left(T e^{\phi}\right)}{\partial r}+\left(T e^{\phi}\right) D_{3} \frac{\partial \eta}{\partial r}\right] .
\end{aligned}
$$




\section{HEAT TRANSPORT AND NEUTRINO DIFFUSION}

The coefficients $D_{2}, D_{3}$, and $D_{4}$ are defined through $(x=\omega / T)$

$$
D_{n}=\int_{0}^{\infty} d x x^{n} D(\omega) f_{0}(\omega)\left(1-f_{0}(\omega)\right)
$$

The explicit expression for the absorption mean free path

$$
\begin{gathered}
\frac{1}{\lambda_{a}}=\frac{G_{F}^{2}}{\pi^{2}} \int_{0}^{\infty} d E_{e} E_{e}^{2}\left[1-f_{e q}\left(E_{e}\right)\right] \int_{-1}^{+1} d \cos \theta \frac{(\cos \theta-1)}{1-e^{-z}}\left[A R_{1}+R_{2}+B R_{3}\right] \\
R_{s}^{\text {out }}=4 G_{F}^{2} \frac{(\cos \theta-1)}{1-e^{-z}}\left[A R_{1}+R_{2}+B R_{3}\right]
\end{gathered}
$$

The polarization functions

$$
\begin{aligned}
& R_{1}=\left(\mathcal{V}^{2}+\mathcal{A}^{2}\right)\left[\operatorname{Im} \Pi_{L}^{R}\left(q_{0}, q\right)+\operatorname{Im} \Pi_{T}^{R}\left(q_{0}, q\right)\right] \\
& R_{2}=\left(\mathcal{V}^{2}+\mathcal{A}^{2}\right) \operatorname{Im} \Pi_{T}^{R}\left(q_{0}, q\right)-\mathcal{A}^{2} \operatorname{Im} \Pi_{A}^{R}\left(q_{0}, q\right) \quad R_{3}=2 \mathcal{V} \mathcal{A} \operatorname{Im} \Pi_{V A}^{R}\left(q_{0}, q\right) .
\end{aligned}
$$

- $\mathcal{V}=C g_{V}$ and $\mathcal{A}=C g_{A}$ for the absorption, $C$ is the Cabibbo factor

- $\mathcal{V}=g_{V} / 2$ and $\mathcal{A}=g_{A} / 2$ for the scattering

To include all six neutrino types, one can redefine the diffusion coefficients

$$
D_{2}=D_{2}^{\nu_{e}}+D_{2}^{D_{e}}, \quad D_{3}=D_{3}^{\nu_{e}}-D_{3}^{D_{e}}, \quad D_{3}=D_{4}^{\nu_{e}}+D_{4}^{D_{e}}+4 D_{4}^{\nu_{\mu}} .
$$

\section{HOT NEUTRON AND QUARK STAR EVOLUTION}

Using the equation for the electron fraction and the matter energy equation

$$
\begin{gathered}
\frac{\partial Y_{e}}{\partial t}=-e^{\phi} \frac{S_{N}}{n_{B}}, \quad Y_{L}=\frac{N_{\nu}}{n_{B}}+Y_{e} \\
\frac{\mathrm{d}\left(E / N_{B}\right)}{\mathrm{d} t}+P \frac{\mathrm{d}\left(V / N_{B}\right)}{\mathrm{d} t}=-e^{\phi} \frac{S_{N}}{n_{B}} \\
T e^{\phi} \frac{\partial \mathbf{s}}{\partial t}+\mu_{\nu} e^{\phi} \frac{\partial \mathbf{Y}_{\mathbf{L}}}{\partial t}+\frac{\partial\left(e^{2 \phi} 4 \pi r^{2} \mathbf{H}_{\nu}\right)}{\partial a}=0
\end{gathered}
$$

we obtain

$$
\begin{gathered}
\frac{\partial N_{\nu} / n_{B}}{\partial t}+\frac{\partial Y_{e}}{\partial t}+\frac{\partial\left(e^{\phi} 4 \pi r^{2} F_{\nu}\right)}{\partial a}=0 \\
\frac{\partial \mathbf{Y}_{\mathbf{L}}}{\partial t}+\frac{\partial\left(e^{\phi} 4 \pi r^{2} \mathbf{F}_{\nu}\right)}{\partial a}=0 \\
F_{\nu}=-\frac{e^{-\Lambda} e^{-\phi} T^{2}}{6 \pi^{2}}\left[\mathrm{D}_{3} \frac{\partial\left(T e^{\phi}\right)}{\partial r}+\left(T e^{\phi}\right) \mathrm{D}_{2} \frac{\partial \eta}{\partial r}\right] \\
H_{\nu}=-\frac{e^{-\Lambda} e^{-\phi} T^{3}}{6 \pi^{2}}\left[\mathrm{D}_{4} \frac{\partial\left(T e^{\phi}\right)}{\partial r}+\left(T e^{\phi}\right) \mathrm{D}_{3} \frac{\partial \eta}{\partial r}\right] .
\end{gathered}
$$




\section{QUASI-EQUILIBRATE EVOLUTION OF COMPACT OBJECT}

The structure equations are

$$
\begin{array}{r}
\frac{\partial r}{\partial a}=\frac{1}{4 \pi r^{2} n_{B} e^{\Lambda}}, \quad \frac{\partial m}{\partial a}=\frac{\rho}{n_{B} e^{\Lambda}} \\
\frac{\partial \phi}{\partial a}=\frac{e^{\Lambda}}{4 \pi r^{4} n_{B}}\left(m+4 \pi r^{3} P\right), \\
\frac{\partial P}{\partial a}=-(\rho+P) \frac{e^{\Lambda}}{4 \pi r^{4} n_{B}}\left(m+4 \pi r^{3} P\right)
\end{array}
$$

with the boundary conditions

$$
\begin{aligned}
r(a=0) & =0 ; \quad m(a=0)=0, \\
\phi\left(a=a_{s}\right) & =\frac{1}{2} \log \left[\begin{array}{c}
2 m\left(a=a_{s}\right) \\
r\left(a=a_{s}\right)
\end{array}\right] ; \quad P\left(a=a_{s}\right)=P_{s}
\end{aligned}
$$

\section{COOLING EVOLUTION WITHOUT NEUTRINO TRAPPING}

- The flux energy per unit time $l(r)$ through a spherical slice at distance $r$ from the center is:

$$
\mathrm{l}(r)=-4 \pi r^{2} \mathbf{k}(r) \underset{\partial r}{\partial\left(\mathbf{T}(r) e^{\Phi}\right)} e^{-\Phi} \sqrt{1-\frac{2 M}{r}}
$$

The factor $e^{-\Phi} \sqrt{1-\frac{2 M}{r}}$ corresponds to the relativistic correction of the time scale and the unit of thickness.

- The equations for energy balance and thermal energy transport are:

$$
\begin{gathered}
\frac{\partial}{\partial N_{B}}\left(l e^{2 \Phi}\right)=-\frac{1}{n}\left(\epsilon_{\nu} e^{2 \Phi}+\mathrm{cv} \frac{\partial}{\partial t}\left(\mathrm{~T} e^{\Phi}\right)\right) \\
\frac{\partial}{\partial N_{B}}\left(\mathrm{~T} e^{\Phi}\right)=-\frac{1}{\mathrm{k}} \frac{\mathrm{l} e^{\Phi}}{16 \pi^{2} r^{4} n}
\end{gathered}
$$

where $n=n(r)$ is the baryon number density, $N_{B}=N_{B}(r)$ is the total baryon number in the sphere with radius $r$ and

$$
\frac{\partial N_{B}}{\partial r}=4 \pi r^{2} n\left(1-\frac{2 M}{r}\right)^{-1 / 2}
$$

D. Blaschke, H. Grigorian and D. N. Voskresensky, Astronomy and Astrophysics 368, 561 (2001). 


\section{STELLAR MATTER UNDER COMPACT STAR CONDITIONS}

The stellar matter including the quark core of compact stars consists of neutrons protons, up, down quarks, electrons and neutrinos in beta equilibrium and with the charge neutrality condition.

- $\beta$-equilibrium $n \longleftrightarrow p+e^{-}+\bar{\nu}_{e} ;-$ in nuclear matter $d \longleftrightarrow u+e^{-}+\bar{\nu}_{e}$-in quark matter

$$
\begin{aligned}
& \mu_{n}=\mu_{p}+\mu_{e}+\mu_{\nu_{e}} \\
& \mu_{d}=\mu_{u}+\mu_{e}+\mu_{\nu_{e}}
\end{aligned}
$$

where $\mu_{e}, \mu_{\nu_{e}}$ are the electrons and the neutrino chemical potentials.

$$
\begin{gathered}
\text { - Charge neutrality } \quad n_{p}-n_{e}=0 \text { : -in nuclear matter } \\
\qquad{ }_{3}^{2} n_{u}-{ }_{3}^{1} n_{d}-n_{e}=0 \text {-in quark matter }
\end{gathered}
$$

The contribution from the leptons should be added to the thermodynamical potential $\Omega_{q}$ :

$$
\left|\Omega\left(\phi, \Delta ; \mu_{q}, \mu_{I}, \mu_{e}, T\right)=\Omega_{q}\left(\phi, \Delta ; \mu_{q}, \mu_{I}, T\right)+\Omega_{e}\left(\mu_{e}, T\right)+\Omega_{\nu_{e}}\left(\mu_{\nu_{e}}, T\right)\right|
$$

\section{CONFIGURATION OF COMPACT STARS WITHOUT SHELL}

\section{CONDENSATE AND TEMPERATURE EFFECT}

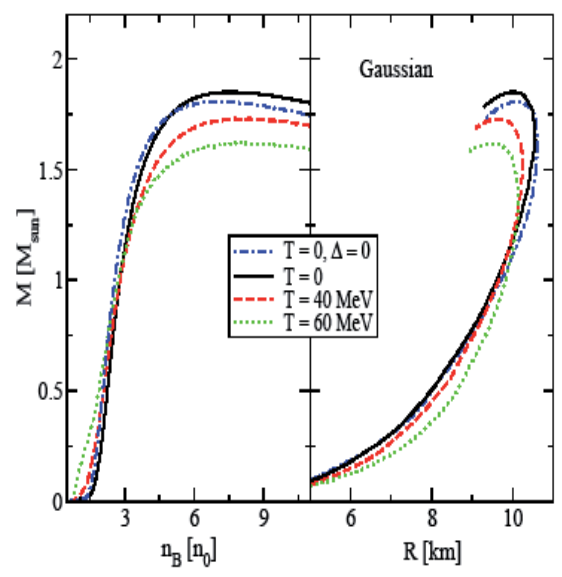

The star configuration could be defined from the Tolman-Oppenheimer-Volkoff equations

$$
\begin{gathered}
\frac{d P}{d r}=-\frac{[\epsilon(r)+P(r)]\left[m(r)+4 \pi r^{3} P(r)\right]}{r[r-2 m(r)]} \\
m=4 \pi \int_{0}^{r} r^{2} \epsilon(r) d r
\end{gathered}
$$

The equations are solved for the set of central densities $n_{q}(0)$ for which the stars are stable. The total mass $M=m(r)$ of the star is defined on the radius $r$ so that $P(r)=0$.

The integral parameters $M$ and $R$ feel the existence of the diquark condensation: the EoS becames softer. Also are sensitive on temperature variations. Both are decresing the values of the $M_{\max }$ and $R_{\max }$ of the core approximately $10 \%$ from their values of the $T=0$ and $\Delta=0$ cases. 


\section{PNS EVOLUTION WITH NEUTRINO TRAPPING}
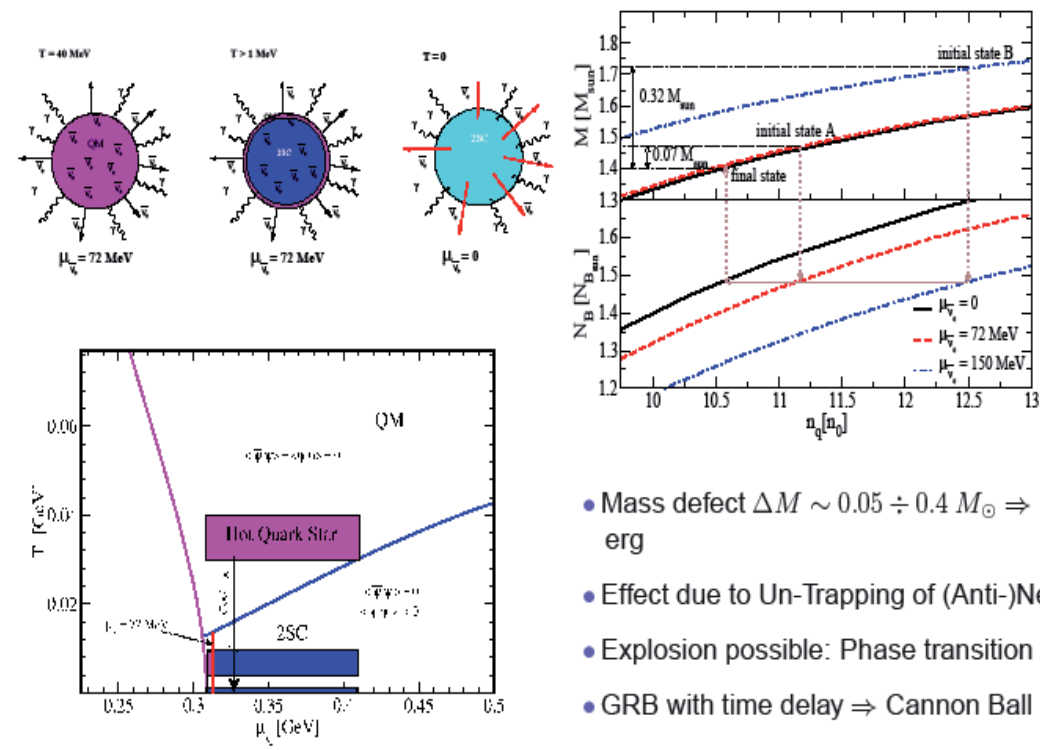

- Mass defect $\Delta M \sim 0.05 \div 0.4 M_{\odot} \Rightarrow 10^{53} \div 10^{54}$ erg

- Effect due to Un-Trapping of (Anti-)Neutrinos - Explosion possible: Phase transition $1^{\text {st }}$ Order - GRB with time delay $\Rightarrow$ Cannon Ball Model

D. N. Aguilera, D. Blaschke, H. Grigorian, arXiv:astro-ph/0212237

\section{MASS DEFECT - ESTIMATION OF ENERGY}

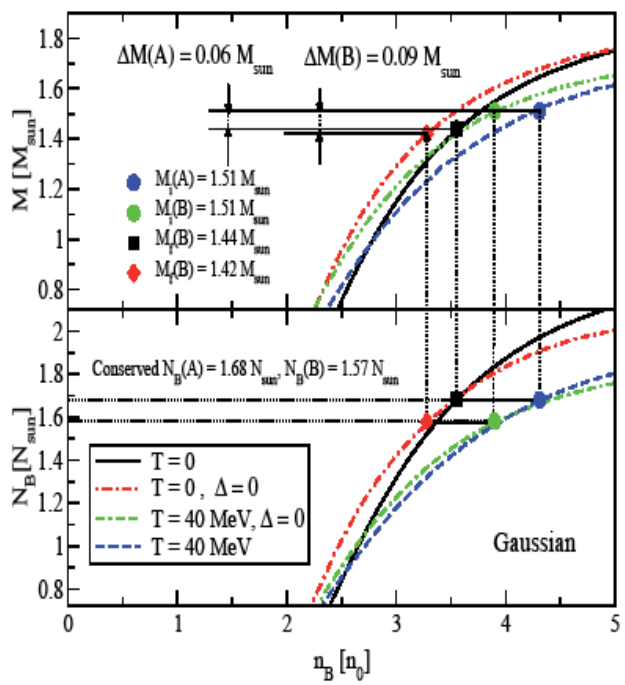

- During the cooling evolution the star must loose an amount of energy: the energy release will have an equivalent in a mass defect. We estimated this from the diquark condensation as about 0.1 $\mathrm{M}_{\odot}$. It corresponds to the estimated energy $(\Delta / \mu)^{2} \simeq 10^{52} \mathrm{erg}$ of the diquark condensate, see Ref. ["].

*D. K. Hong, S. D. Hsu and F. Sannino, Phys. Lett. B516,362 (2001) 


\section{COOLING OF HYBRID NEUTRON STARS}

\section{LECTURE 2}

- Configurations of Hybrid stars

- Cooling processes in QM

- Crust, SC Gaps and ...

- Time Evolution of Surface Temperature

- Constraints from Observations

○ Summary \& Discussion

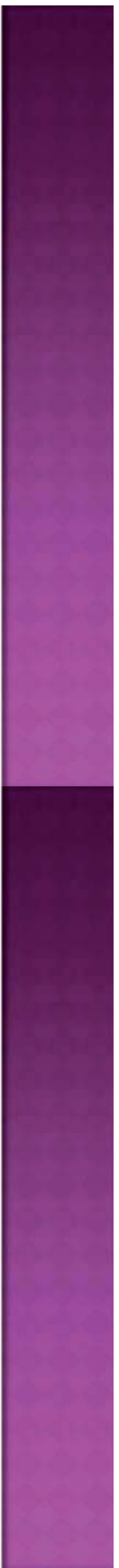




\section{PHASE TRANSITION: \\ NUCLEAR MATTER $\longrightarrow$ QUARK MATTER}

- Walecka model $(\mathrm{p}, \mathrm{n}, \mathrm{e})+(\sigma, \omega) \longrightarrow$ Bag model $(\mathrm{u}, \mathrm{d}, \mathrm{e})$

- Maxwell construction: $P^{H a d}=P^{Q u a r k} \quad \mu_{B}^{\text {Had }}=\mu_{B}^{\text {Quark }}$
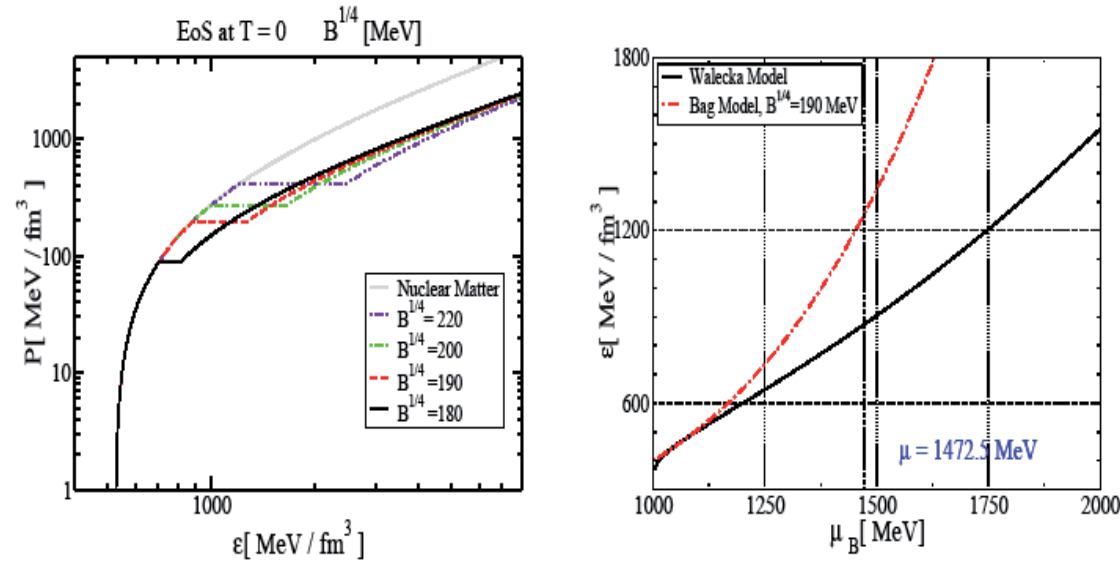

\section{STABILITY OF HADRONIC AND HYBRID STARS}
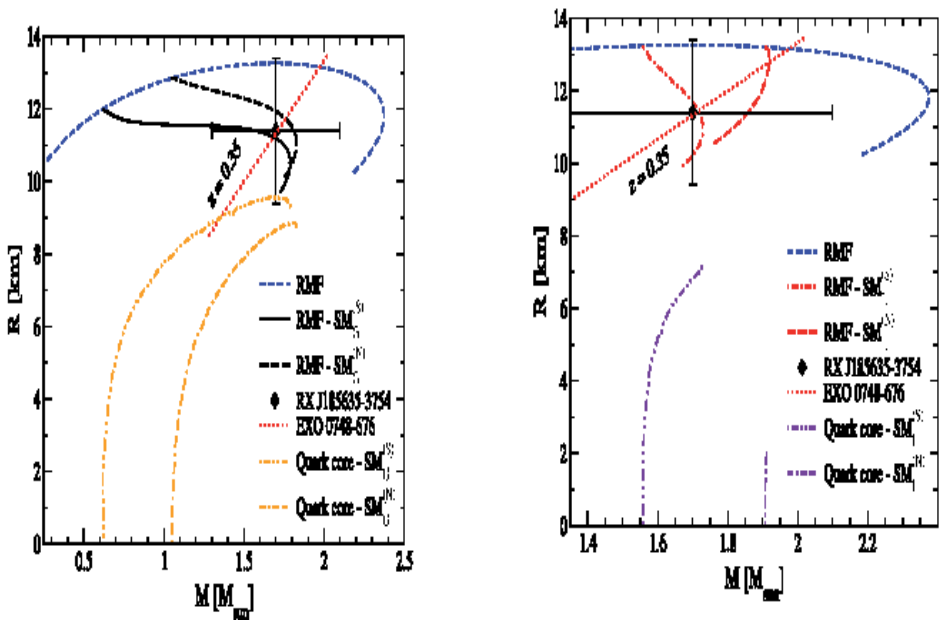

HOVIK GRIGORIAN 


\section{MYBRID CONFUGURATIONS}

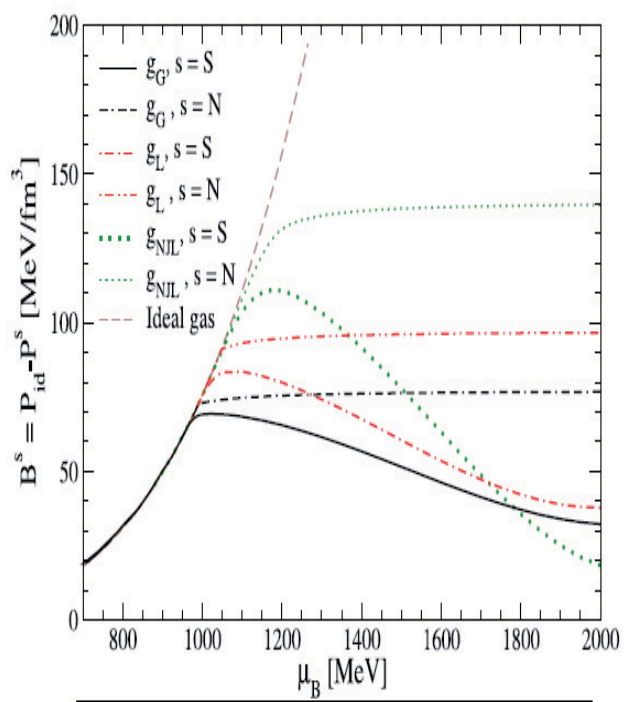

- Critical masses for phase transition $M_{c}=1.2 M_{\odot} \mathrm{HHJ}$ - INCQM (Gaussian)with $2 \mathrm{SC}$

$M_{c}=1.82 M_{\odot}$ - without 2SC

H. Grigorian, D. Blaschke, D.N. Aguilera. Phys.Rev.C 69 (2004) 065802

\section{COOLING MECHANISM}

\section{Neutrino processes in quark matter: Emissivities}

3 Quark direct Urca (QDU) the most efficient processes

$d \rightarrow u+e+\bar{\nu}$ and $u+e \rightarrow d+\nu$

$\epsilon_{\nu}^{\mathrm{QDU}} \simeq 9.4 \times 10^{26} \alpha_{s} u Y_{e}^{1 / 3} \zeta_{\mathrm{QDU}} T_{9}^{6} \mathrm{erg} \mathrm{cm}^{-3} \mathrm{~s}^{-1}$,

Compression $u=n / n_{0} \simeq 2$, strong coupling $\alpha_{s} \approx 1$

$\Rightarrow$ Quark Modified Urca (QMU) and Quark Bremsstrahlung (QB)

$d+q \rightarrow u+q+e+\bar{\nu}$ and $q_{1}+q_{2} \rightarrow q_{1}+q_{2}+\nu+\bar{\nu}$

$\epsilon_{\nu}^{\mathrm{QMU}} \sim \epsilon_{\nu}^{\mathrm{QB}} \simeq 9.0 \times 10^{19} \zeta_{\mathrm{QMU}} T_{9}^{8} \mathrm{erg} \mathrm{cm}^{-3} \mathrm{~s}^{-1}$.

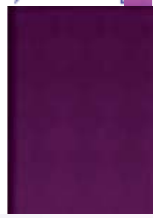

$\rightarrow$ Suppression due to the pairing (2CS, CFL, $\mathbf{X}$ )

$\mathrm{QDU}: \zeta_{\mathrm{QDU}} \sim \exp \left(-\Delta_{q} / T\right)$

QMU and $\mathrm{QB}: \zeta_{\mathrm{QMU}} \sim \exp \left(-2 \Delta_{q} / T\right)$ for $T<T_{\text {crit }, q} \simeq 0.4 \Delta_{q}$

$\Rightarrow \mathbf{P B F} e+e \rightarrow e+e+\nu+\bar{\nu}$

$\epsilon_{\nu}^{e e}=2.8 \times 10^{12} Y_{e}^{1 / 3} u^{1 / 3} T_{9}^{8} \mathrm{erg} \mathrm{cm}^{-3} \mathrm{~s}^{-1}$,

becomes important for $\Delta_{q} / T>>1$

D. Blaschke, H. Grigorian, D.N. Voskresensky, Astron. \& Astrophys. 368 (2001) 561 


\section{DIRECT URCA IN UADRON MAT『ER}

- DU critical densities

$n_{c}=2.7 n_{0}$ non linear Walecka (NLW) $n_{c}=5.0 n_{0}$ Heiselberg, Hjorth-Jenson

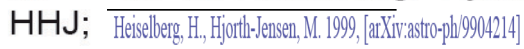
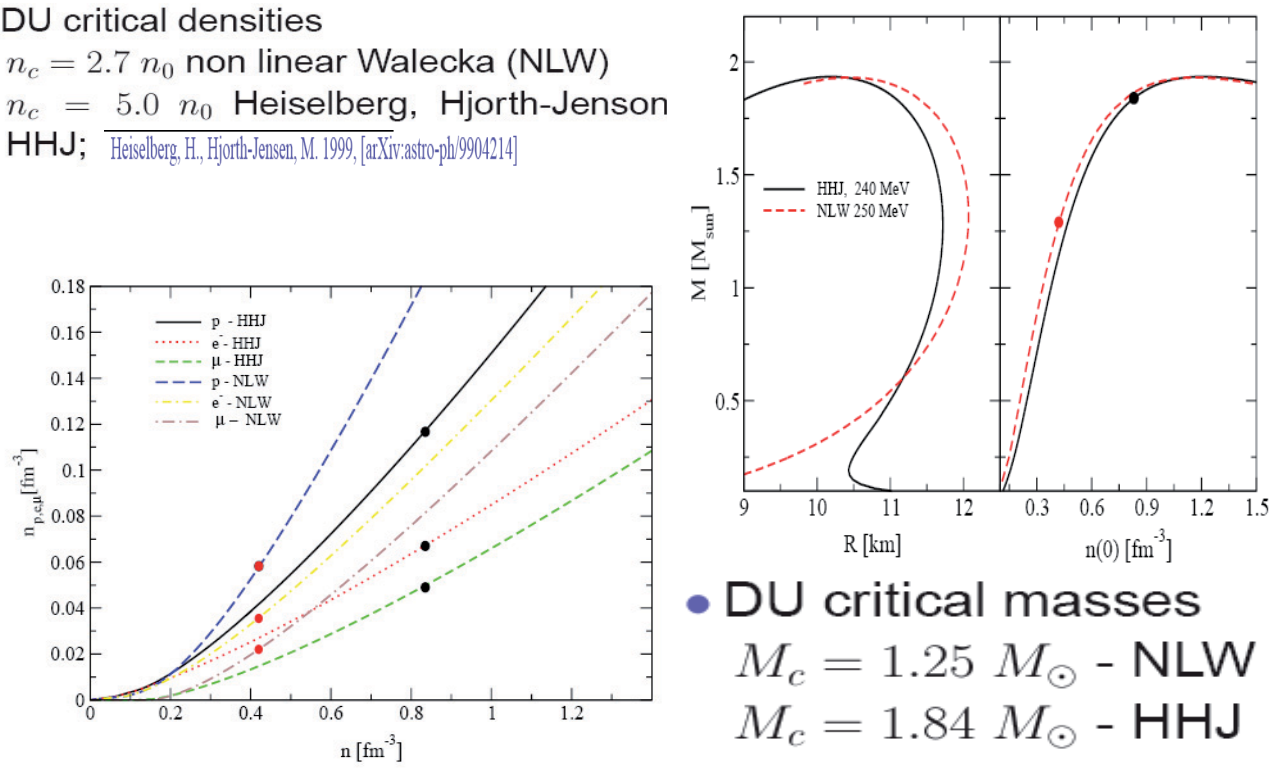

- DU critical masses

$$
\begin{aligned}
& M_{c}=1.25 M_{\odot}-\mathrm{NLW} \\
& M_{c}=1.84 M_{\odot}-\mathrm{HHJ}
\end{aligned}
$$

\section{DU CONSTRANNT}

$n \rightarrow p+e+\bar{\nu}_{e}$ implies $p_{n} \leq p_{p}+p_{e}$, charge neutrality results in

$$
x_{D U}\left(x_{e}\right) \geq \frac{1}{1+\left(1+x_{e}^{1 / 3}\right)^{3}}
$$

$\Rightarrow$ no muons:

$x_{D U}=11.1 \%$

$\Rightarrow$ relativistic limit $\left(n_{e}=n_{\mu}\right): x_{D U}=14.8 \%$

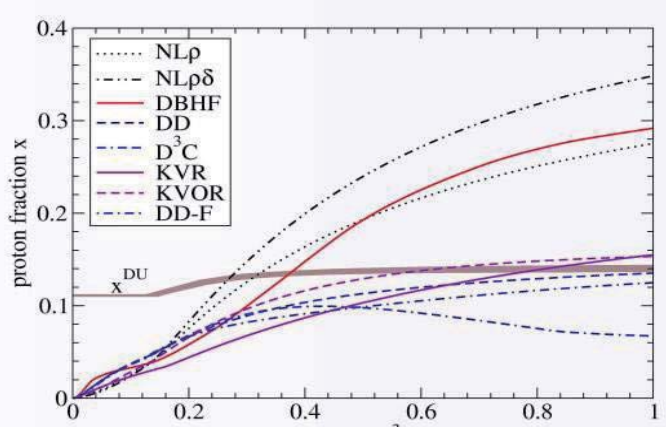

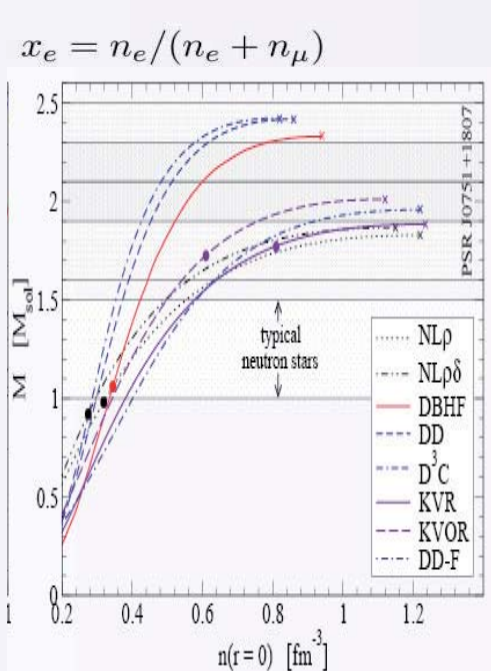




\section{HS WITH DIFFERENT STRUCTURE}
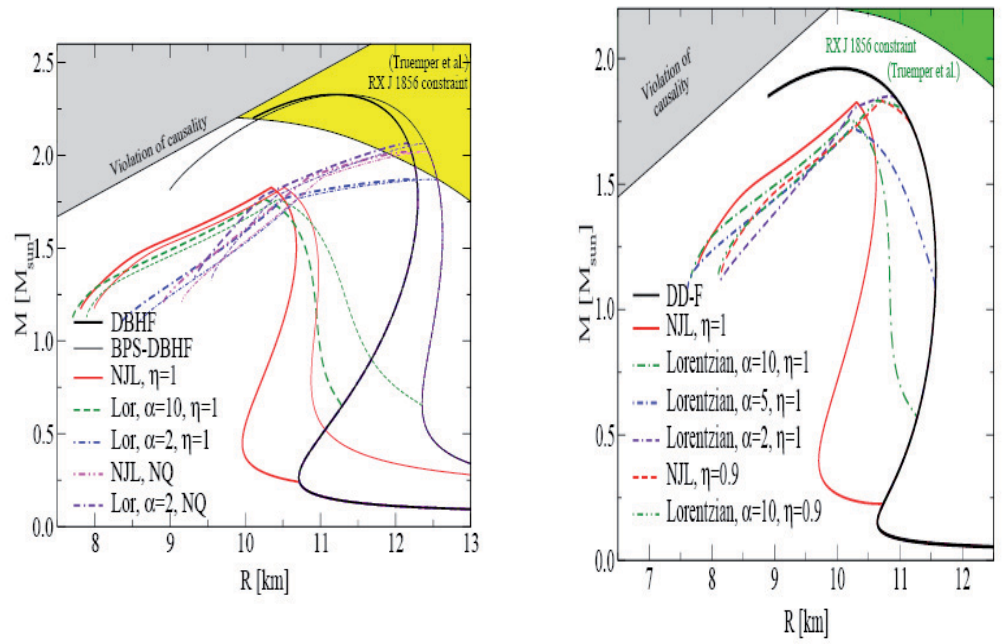

\section{SC PARNNG GAPS}

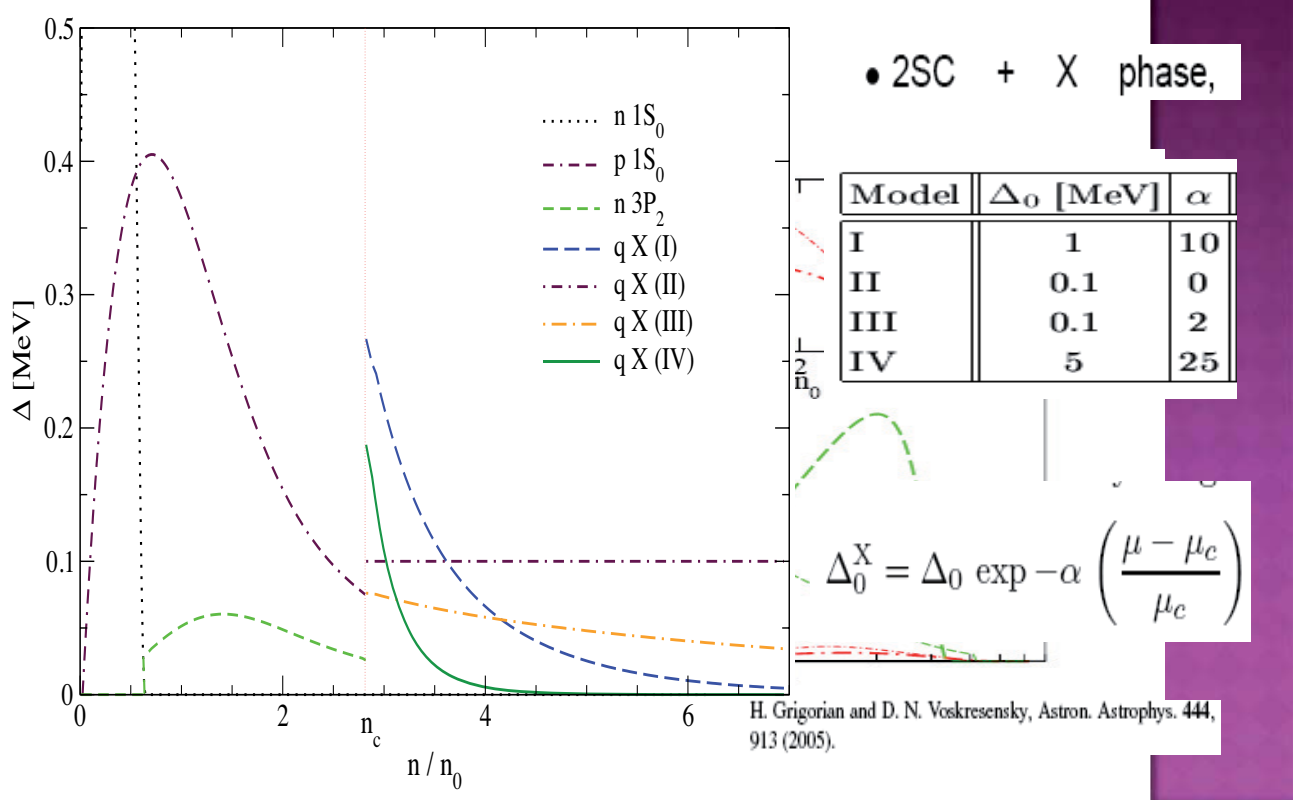




\section{CRUST MODEL}

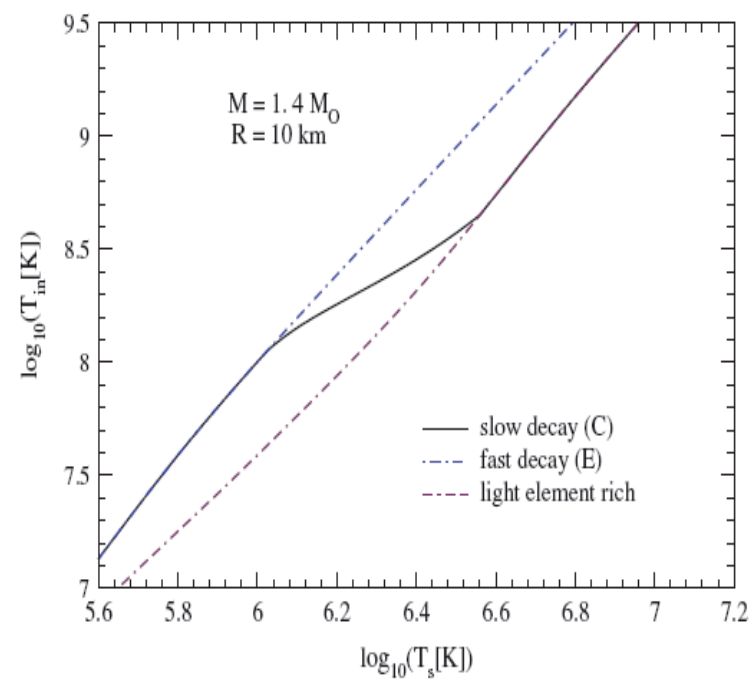

$$
\Delta M_{\mathrm{L}}(t)=e^{-t / \tau} \Delta M_{\mathrm{L}}(0)
$$

D. Page, J. M. Lattimer, M. Prakash, and A. W. Steiner, Astrophys. J. Supp. 155, 623 (2004).

D. G. Yakovlev, K. P. Levenfish, A. Y. Potekhin, 0. Y. Gnedin, and G. Chabrier, Astron. Astrophys. 417, 169 (2004).

\section{SURFACE TEMPERATURE \& AGE DATA}

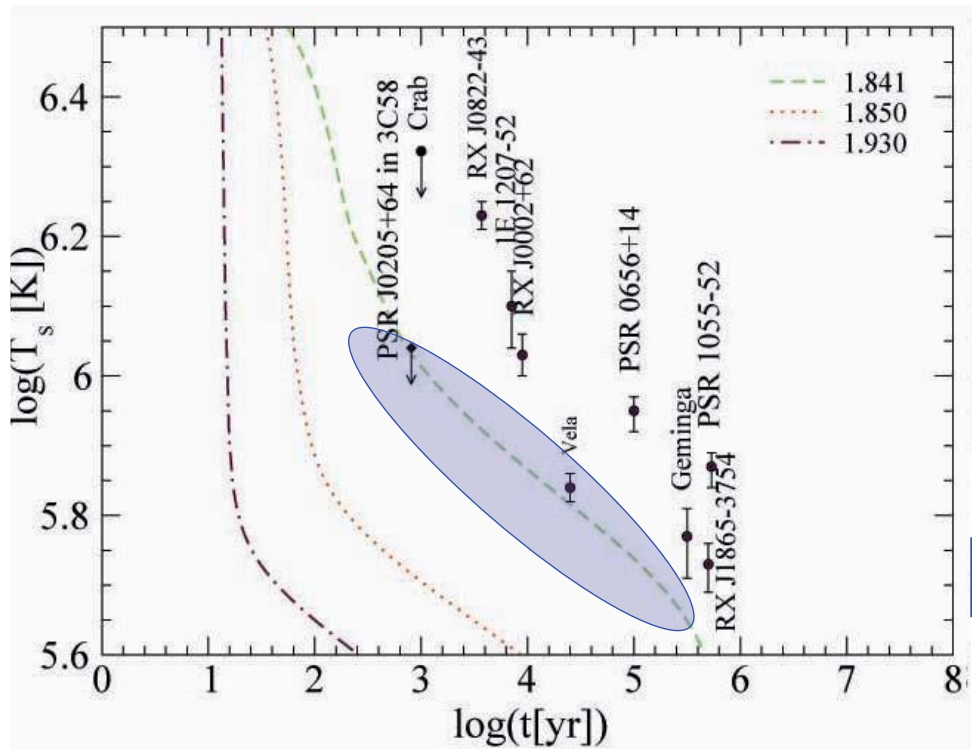

$\frac{d U}{d t}=\sum_{i} C_{i} \frac{d T}{d t}=-\varepsilon_{\gamma}-\sum_{j} \varepsilon_{\nu}^{j}$

Data taken from:

Yakovlev et al.,

A \& A 389 (2002) L24;

Medium Cooling Scenario:

D. Blaschke, H. Grigorian and D. N. Voskresensky, A\&A 424 (2004) 979.

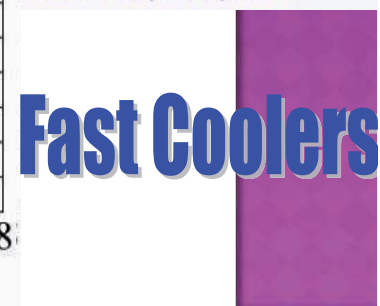




\section{COOLNG OF UIII STARS MODELS}

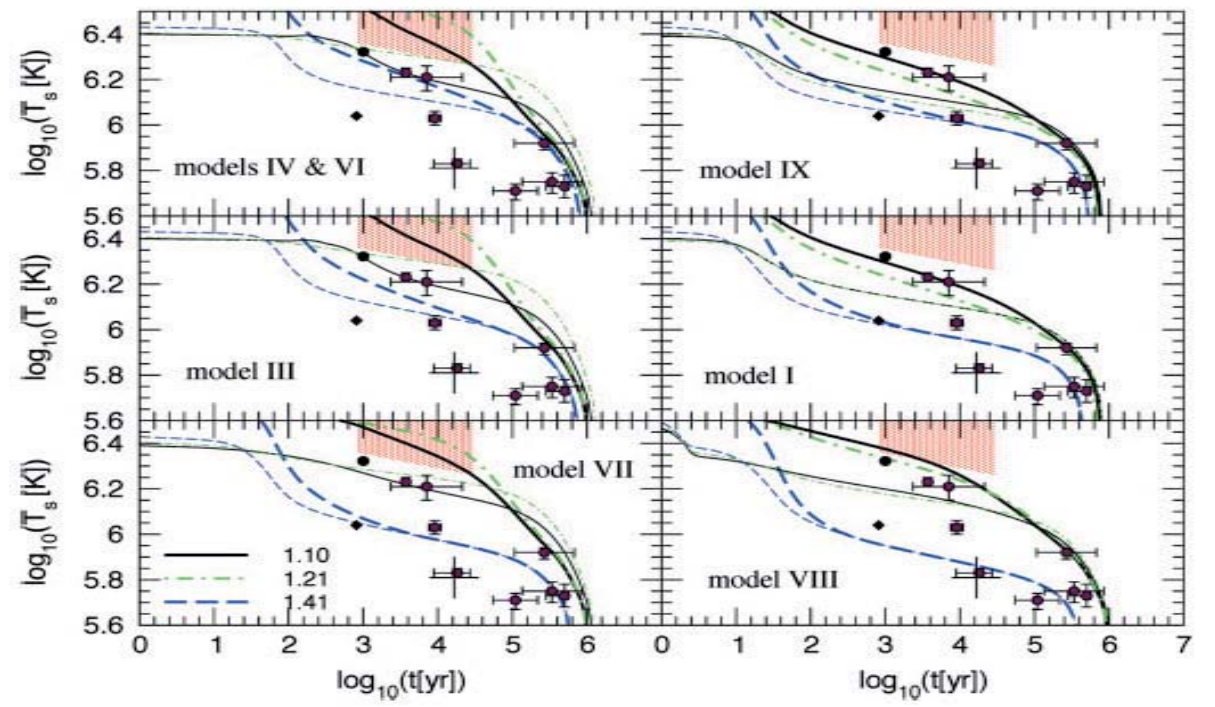

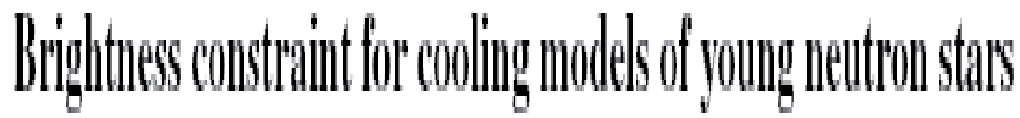

(i) All objects with temperatures exceeding $T=4 \times 10^{6} \mathrm{~K}$ are potentially observable. The probability of finding an object increases with its luminosity because of the increase of the visibility volume and reduction of the absorption of $\mathrm{x}$-rays by the interstellar medium

(ii) The distribution of young objects is a steplike function of temperature for higher temperatures.

(iii) The uncertainty of the $B C$ border is of the same order as the error bars of the measured temperatures at the corresponding age.

H. Grigorian, Phys. Rev. G74:025801,2006 


\section{SOME ANOMALIES}

The most of pathologies of hadronic stars are descendible

- with AV18 gaps, $\pi$-condensate, MMU - with Gaps from Yakovlev at al. 2003 ( $3 P_{2}$ - is NOT suppressed)
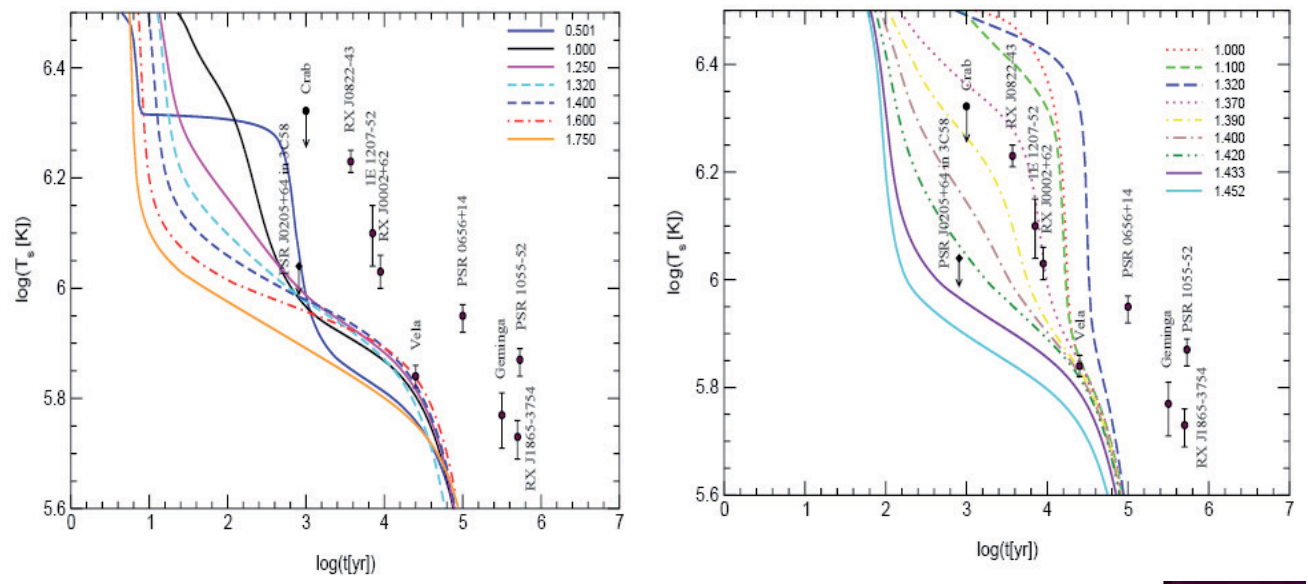

\section{WHY DU IS A PROBLEM?}
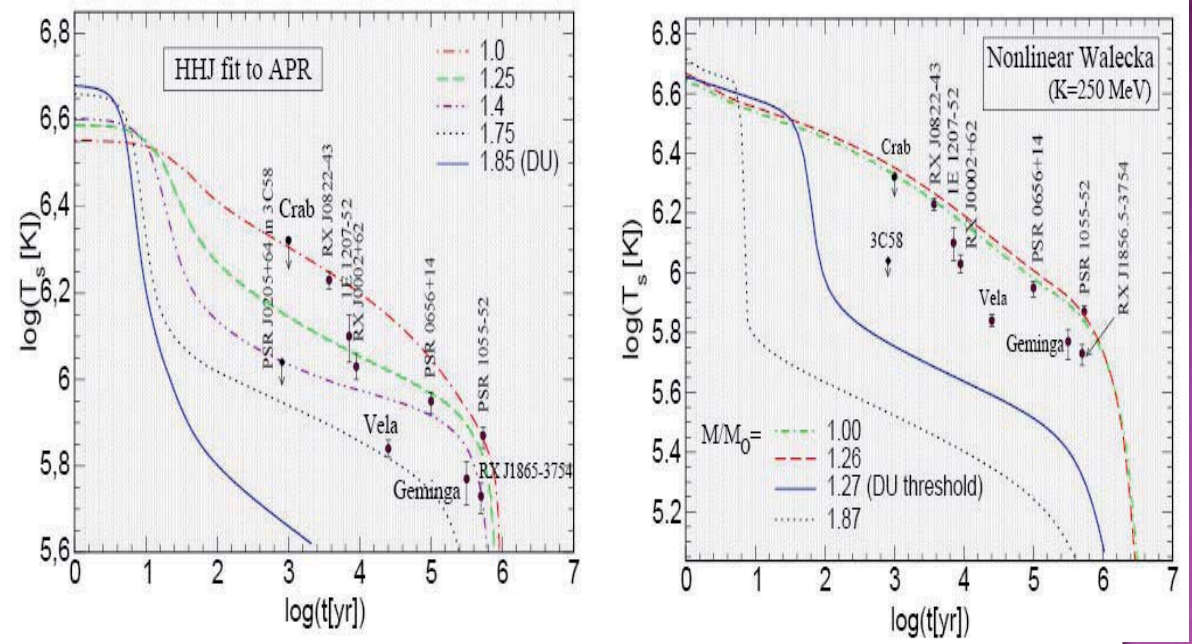


\section{TEMPERATUR 听 TUE STAR UNTERIOR}
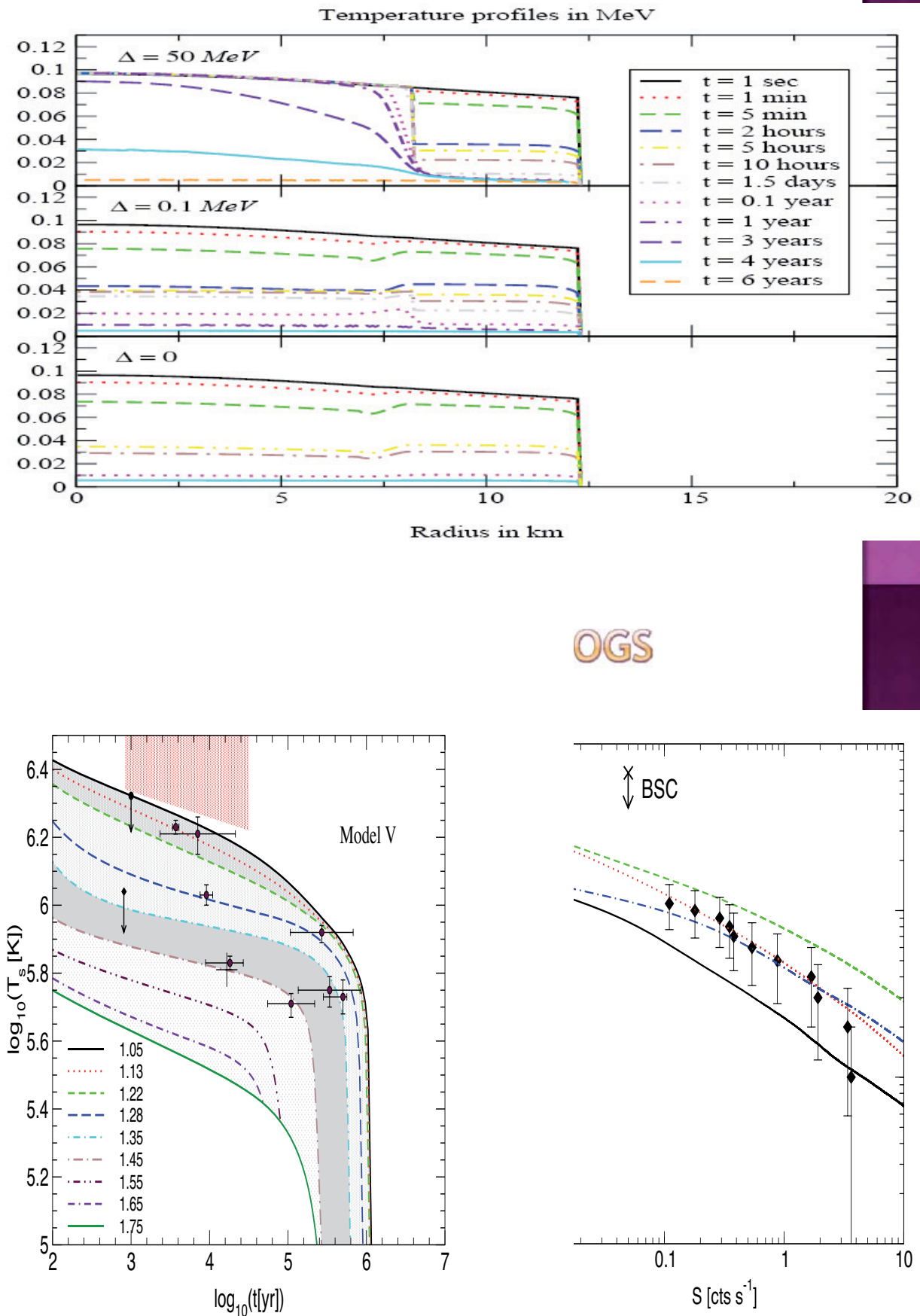


\section{COOLNAG OF MS WITH SINGLE FLAVOR QM}

\section{Hybrid stars with d-quark drip}

$\odot$
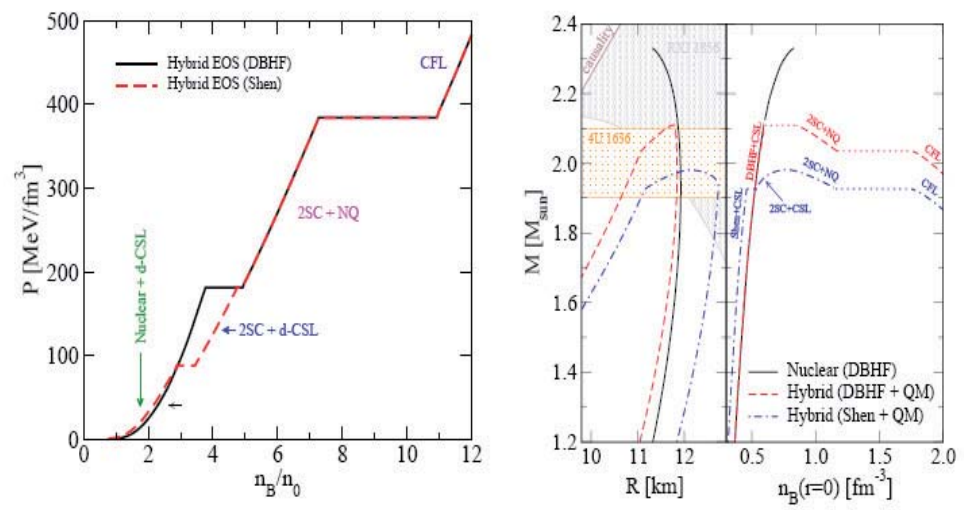

D. Blaschke, F.S., T. Klähn, in preparation
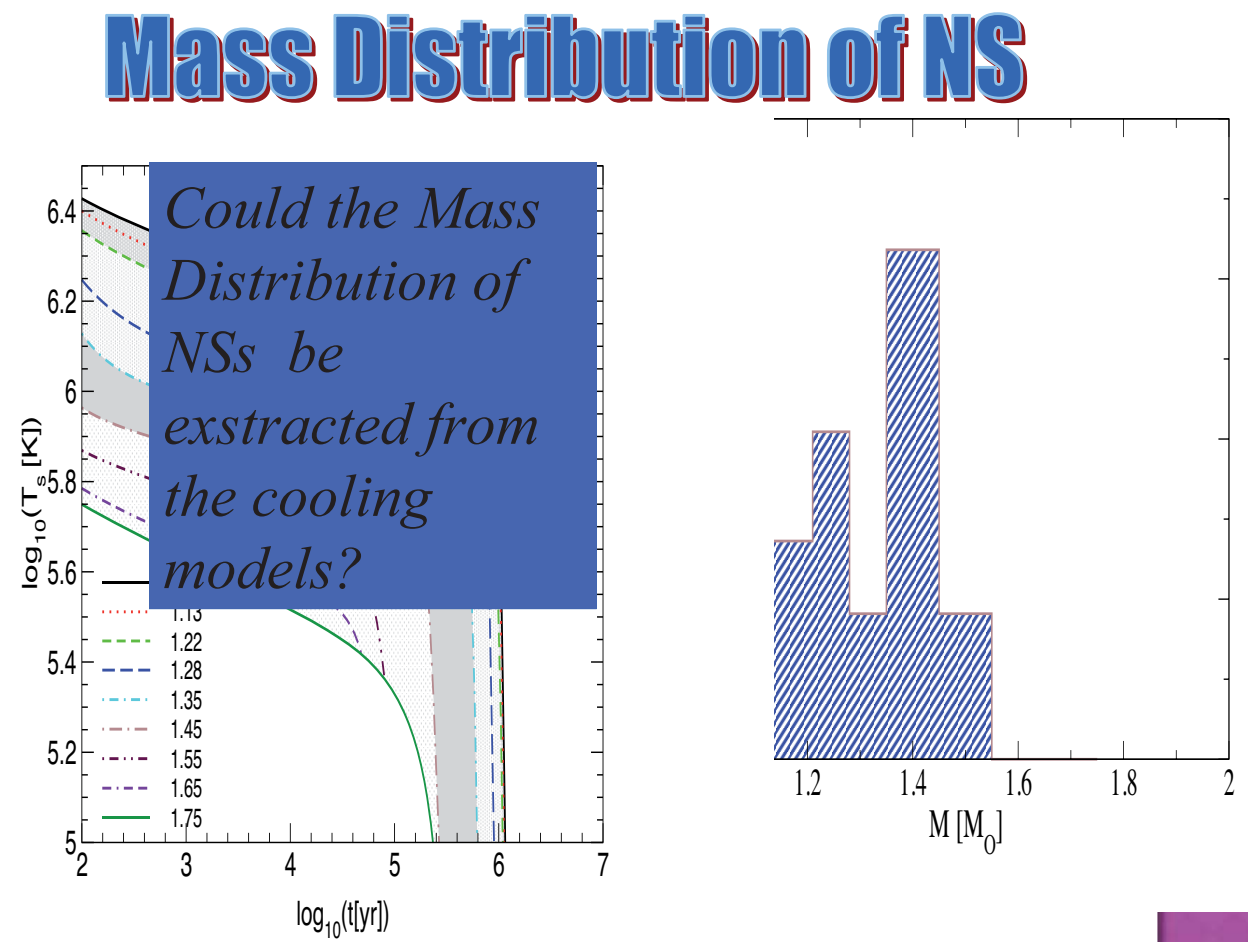


\section{EXTRACTED MASS DISTRRBUTION}

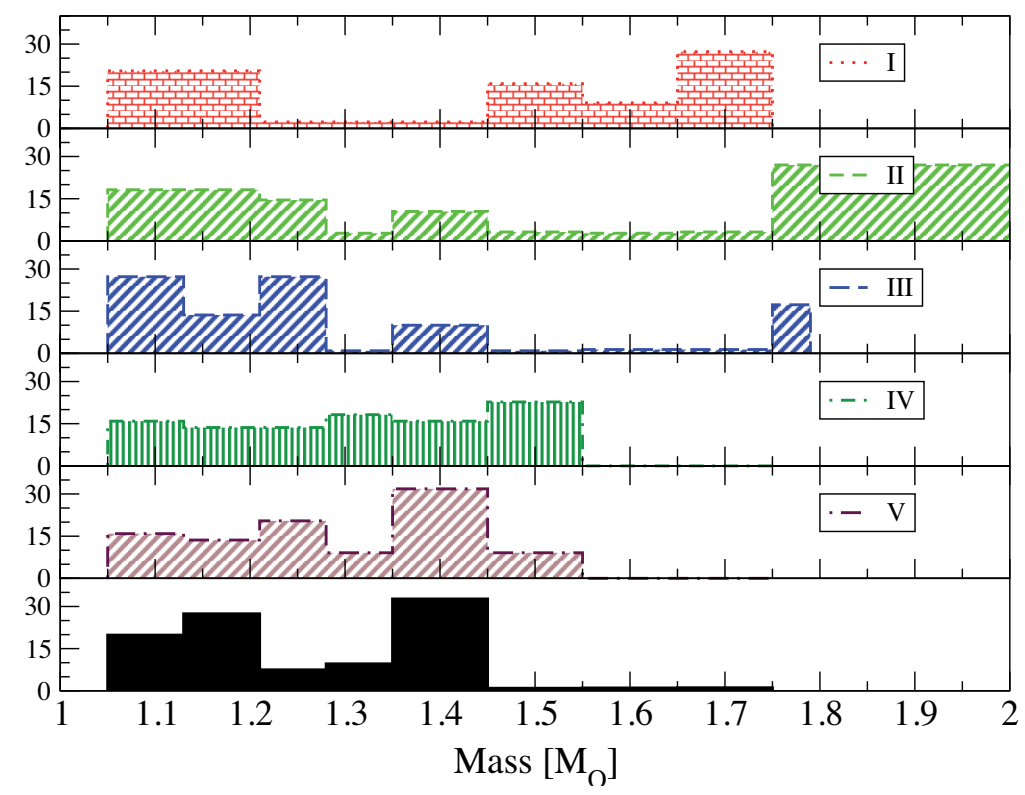

\section{SUMMARY}

- Cooling of NSs is one of testing schemes for High density matter EoS

- Mass distribution of NS is extracted from the cooling model

- Cooling data allowed existence of HS even with one flaver QM

$\odot$ DU can be consided as a problem 


\section{OPEN QUESTIONS \& DISCUSSIAN}

$\odot$ Do Hybrid NSs an alternative or the solution of problems of compact stars?

- Do for all constraints exist a valid HNS model?

- Can magnetic field and surface heating modify the main conclusions on cooling of NS?

○ Is the mass distribution of NSs an obsorvable? 